\title{
EXACT SOLUTION OF A VERTEX MODEL WITH UNLIMITED NUMBER OF STATES PER BOND
}

\author{
F. C. Alcaraz ${ }^{1}$ and R. Z. Bariev ${ }^{1,2}$ \\ ${ }^{1}$ Departamento de Física, Universidade Federal de São Carlos, 13565-905, São Carlos, SP Brazil \\ ${ }^{2}$ The Kazan Physico-Technical Institute of the Russian Academy of Sciences, Kazan 420029, \\ Russia
}

\begin{abstract}
The exact solution is obtained for the eigenvalues and eigenvectors of the row-to-row transfer matrix of a two-dimensional vertex model with unlimited number of states per bond. This model is a classical counterpart of a quantum spin chain with an unlimited value of spin. This quantum chain is studied using general predictions of conformal field theory. The long-distance behaviour of some ground-state correlation functions is derived from a finite-size analysis of the gapless excitations.
\end{abstract}

Published in J.Phys.A: 32 L25 (1999)

PACS numbers: 75.10.Lp, 74.20-z, 71.28+d 
Most of exactly solved models in two dimensions are known to be related to the six-vertex model solved by Lieb [1]. This model exhibits an infinite number of conserved integrals commuting with each other. The generator of these integrals of motion is the row-to-row transfer matrix. The conserved quantities are quantum Hamiltonians describing different one-dimensional systems, in particular one of these describes the XXZ chain [2]. There exist also exact solutions for a variety of generalizations of this model. In particular lattice models where the link variables may assume $q>2$ different states were solved using the Bethe-anstaz method [3-12].

In this letter we present an exactly integrable vertex model in which the number of different states of a given link is not limited, and may be considered as a generalization of the six-vertex model for the case where on each link of the lattice we may have an unlimited number of lines. This model is the classical counterpart of a quantum spin chain with unlimited value of the spin. The vertex model may be formulated as follows. We consider a square lattice with $M$ rows and $N$ columns and with periodic boundary conditions. At the bonds connecting adjacent pair of lattice sites we attach a variable $\alpha=0,1,2, \ldots$, representing the number of lines (or particles) in the bond. The links $(\alpha, \beta, \gamma, \delta=0,1,2, \ldots)$ connected with a given site produce a vertex configuration, with Boltzmann weight $R_{\gamma, \delta}^{\alpha, \beta}$ as in figure 1. The model we consider obey a type of "ice condition" [1], where the Boltzmann weights are chosen such that the number of lines is conserved as we go from the direction $(\alpha, \beta)$ to $(\gamma, \delta)$, i. e., $\alpha+\beta=\gamma+\delta$. The non-zero Boltzmann weights are given by

$$
\begin{aligned}
& R_{0,0}^{0,0}=a, \quad R_{0,1}^{0,1}=R_{1,0}^{1,0}=b, \\
& R_{1,0}^{0,1}=R_{0,1}^{1,0}=c
\end{aligned}
$$

and

$$
\begin{aligned}
R_{1,1}^{0,2} & =R_{0,2}^{1,1}=R_{n, m+1}^{m, n+1}=b, \quad R_{n, 0}^{n, 0}=b^{n} / a^{n-1}, \\
R_{n+l, m}^{m+l, n} & =\frac{c^{2}}{a}\left(\frac{b}{a}\right)^{l}, \quad R_{l, m}^{m+l, 0}=R_{m+l, 0}^{l, m}=c\left(\frac{b}{a}\right)^{l},
\end{aligned}
$$

where $m, n>0, l \geq 0$ and $a, b, c$ are arbitrary parameters. For convenience we use the 
Baxter's parametrization [3]

$$
a=\sinh \frac{1}{2}(\lambda-u), \quad b=\sinh \frac{1}{2}(\lambda+u), \quad c=\sinh \lambda
$$

where $u$ and $\lambda$ are the spectral and crossing parameters, respectively. If we exclude in (1)-(2) the configurations where $\alpha, \beta, \gamma, \delta>1$ we obtain the standard six-vertex model [3].

To calculate the partition function of the system we use the row-to-row transfer matrix $T$, with elements $T_{\{\alpha\},\left\{\alpha^{\prime}\right\}}$, given by the product of the Boltzmann weights connecting two sucessive rows $\left\{\alpha_{1}, \alpha_{2}, \ldots, \alpha_{N}\right\}$ and $\left\{\alpha_{1}^{\prime}, \alpha_{2}^{\prime}, \ldots, \alpha_{N}^{\prime}\right\}$

$$
T(u)_{\{\alpha\},\left\{\alpha^{\prime}\right\}}=\sum_{\beta_{1}, \beta_{2}, \ldots, \beta_{N}=1}^{\infty} R_{\alpha_{1}^{\prime}, \beta_{N}}^{\alpha_{1}, \beta_{1}}(u) R_{\alpha_{2}^{\prime}, \beta_{1}}^{\alpha_{2}, \beta_{2}}(u) \ldots R_{\alpha_{N}^{\prime}, \beta_{N-1}}^{\alpha_{N}, \beta_{N}}(u) .
$$

The above transfer matrices with different values of the spectral parameter commute among themselves, i. e.,

$$
T(v) T(u)=T(u) T(v) .
$$

A sufficient condition for this commutativity is the existence of $X_{\gamma, \delta}^{\alpha, \beta}\left(u, u^{\prime}\right)$ satisfying the Yang-Baxter equations [3]

$$
\sum_{\gamma, \delta^{\prime \prime}, \mu^{\prime \prime}} R_{\alpha, \delta}^{\gamma, \delta^{\prime \prime}}(u) R_{\gamma, \mu}^{\beta, \mu^{\prime \prime}}\left(u^{\prime}\right) X_{\delta^{\prime \prime}, \mu^{\prime \prime}}^{\mu^{\prime}, \delta^{\prime}}\left(u, u^{\prime}\right)=\sum_{\gamma, \delta^{\prime \prime}, \mu^{\prime \prime}} X_{\delta, \mu}^{\mu^{\prime \prime}, \delta^{\prime \prime}}\left(u, u^{\prime}\right) R_{\alpha, \delta^{\prime \prime}}^{\gamma, \delta^{\prime}}\left(u^{\prime}\right) R_{\gamma, \mu^{\prime \prime}}^{\beta, \mu^{\prime}}(u)
$$

Although we did not succeed in finding $X_{\gamma, \delta}^{\alpha, \beta}\left(u, u^{\prime}\right)$ we have checked equation (5) directly. We want to solve the eigenvalue equation of the transfer matrix

$$
T \Psi=\Lambda \Psi
$$

An obvious way to describe the state of a row is to specify the positions of individual particles (lines). Let

$$
\Phi\left(x_{1}, x_{2}, \ldots, x_{n}\right)
$$

be the amplitude corresponding to the state of a row with particles (lines) at the bonds $x_{1}, x_{2}, \ldots, x_{n}$. Unlike the ordinary six-vertex model, some of $x_{i}$ may coincide 


$$
x_{1} \leq x_{2} \leq \ldots \leq x_{n}
$$

A possible way to represent the model under consideration is to draw as much lines on an edge as the value of the variable $\alpha$ (number of particles) of this edge. An example of arrangement of lines is shown in figure 2. From the definition of our model (1-2) and Fig. 2 it is clear that as in the ordinary six-vertex model [1] the lines link together by forming continuous noncrossing paths through the lattice. If one starts by following a path upwards or to the right, then one will always be travelling in one or other of these two directions, never downwards or to the left. The cyclic boundary conditions ensure that a path never ends. A typical configuration of lines in adjacent rows is shown in Fig. 3. Consequently the total number of particles (lines) on arrow is a conserved quantity and our model can be treated similarly as in $[1,3]$. However in our case the calculation is more complicated. We write the amplitude in (8) in the form of the Bethe ansatz $[3,4]$

$$
\Phi\left(x_{1}, \ldots, x_{n}\right)=\sum_{P} A_{P_{1} \ldots P_{n}} \exp \left(i k_{P_{1}} x_{1}+\ldots+i k_{P_{n}} x_{n}\right),
$$

where the sum is over all the permutations $P$ of $(1,2, \ldots, n)$ and $k_{i}(i=1,2, \ldots, n)$ are unknown quasimomenta. The eigenvalues $\Lambda_{\{k\}}$ corresponding to the eigenvector $\Psi_{\{k\}}$ with amplitudes (10) are given by

$$
\Lambda_{\{k\}}=a^{N} \prod_{j=1}^{n} L\left(k_{j}\right)+b^{N} \prod_{j=1}^{n} N\left(k_{j}\right),
$$

where $L(k)$ and $N(k)$ can be calculated by considering a single particle $n=1$. In our case we have

$$
\begin{aligned}
L(k) & =\left[a b+\left(c^{2}-b^{2}\right) e^{i k}\right] /\left[a^{2}-a b e^{i k}\right], \\
N(k) & =e^{i k}\left[1-c^{2}\left(a^{2}-a b e^{i k}\right)^{-1}\right] .
\end{aligned}
$$

Considering the eigenvalue equations at the boundaries of the inequalites (9) after a long, but straightforward calculation, we obtain the conditions for the coefficients $A_{P_{1} \ldots P_{n}}$,

$$
A_{\ldots P_{1} P_{2} \ldots}=-\left(1-2 \Delta e^{i k_{P_{1}}}+e^{i k_{P_{1}}+i k_{P_{2}}}\right)
$$




$$
\begin{aligned}
\cdot & \left(1-2 \Delta e^{i k_{P_{2}}}+e^{i k_{P_{1}}+i k_{P_{2}}}\right)^{-1} e^{i k_{P_{2}}-i k_{P_{1}}} A_{\ldots P_{2} P_{1} \ldots}, \\
A_{P_{1} \ldots P_{n}}= & A_{P_{2} \ldots P_{n} P_{1}} \exp \left(i k_{P_{1}} N\right)
\end{aligned}
$$

where

$$
\Delta=\left(a^{2}+b^{2}-c^{2}\right) / 2 a b=-\cosh \lambda .
$$

The Bethe-ansatz equations that come from (13)-(15) and fix the values of $\{k\}$ are simplified by using the standard parametrization of the six-vertex model [3]

$$
e^{i k_{j}}=\frac{\sinh \frac{1}{2}\left(\lambda-u_{j}\right)}{\sinh \frac{1}{2}\left(\lambda+u_{j}\right)}
$$

and are given by

$$
\left[\frac{\sinh \frac{1}{2}\left(\lambda+u_{j}\right)}{\sinh \frac{1}{2}\left(\lambda-u_{j}\right)}\right]^{N+n}=(-1)^{n-1} e^{-i P} \prod_{l=1}^{n} \frac{\sinh \left[\lambda+\frac{1}{2}\left(u_{j}-u_{l}\right)\right.}{\sinh \left[\lambda-\frac{1}{2}\left(u_{j}-u_{l}\right)\right.}
$$

where

$$
P=\sum_{l=1}^{n} k_{l}(\bmod 2 \pi)
$$

is the associated momentum of the eigenstate $\Psi_{\{k\}}$. The eigenvalue (11) is now given by

$$
\Lambda(u)_{\{k\}}=a^{N} \prod_{j=1}^{n} \tilde{L}\left(u_{j}\right)+b^{N}\left(\frac{b}{a}\right)^{n} \exp (i P) \prod_{j=1}^{n} \tilde{M}\left(u_{j}\right)
$$

where

$$
\begin{aligned}
\tilde{L}\left(u_{j}\right) & =\frac{\sinh \left[\lambda+\frac{1}{2}\left(u-u_{j}\right)\right]}{\sinh \frac{1}{2}\left(u_{j}-u\right)} \\
\tilde{M}\left(u_{j}\right) & =\frac{\sinh \left[\lambda-\frac{1}{2}\left(u-u_{j}\right)\right]}{\sinh \frac{1}{2}\left(u-u_{j}\right)} .
\end{aligned}
$$

Equations (10)-(18) completely describe the eigenvectors and eigenvalues of the row-to-row transfer matrix of the model with Boltzmann weights (1)-(2). From the comparison of these equations with the corresponding equations of the six-vertex model it is clear that the eigenvalues of the transfer matrix of the model under consideration up to the multiplicative 
factor $a^{n}$ coincide with the eigenvalues of the transfer matrix of a six-vertex model with $N+n$ columns but with Boltzmann weights modified along a vertical "seam" in the lattice $[13,14]$. The boundary angle, that modifies the Boltzmann weights along the seam is equal to the momentum $P$ of the state.

Let us consider the thermodynamic limit where $n \rightarrow \infty$ and $N \rightarrow \infty$, but the density of particles (or density of lines) $\rho=n / N$ is kept fixed. The equations (16)-(17) can be rewritten in terms of integral equations. In the region $-1 \leq \Delta<1, \lambda=i \mu, 0 \leq \mu<\pi$ we have

$$
\begin{gathered}
2 \pi \rho(u)+\int_{-u_{0}}^{u_{0}} \frac{\sin (2 \mu) \rho\left(u^{\prime}\right)}{\cosh \left(u-u^{\prime}\right)-\cos (2 \mu)} d u^{\prime}=\frac{\sin \mu}{\cosh u-\cos \mu}(1+\rho), \\
\int_{-u_{0}}^{u_{0}} \rho(u) d u=\rho, \\
\frac{1}{N} \ln [\Lambda(u)]=-\rho \ln a+(1+\rho) \max \left\{\ln a+\int_{-u_{0}}^{u_{0}} \ln [\tilde{L}(u)] \rho(u) d u\right. \\
\left.\ln b+\frac{i P}{N}+\int_{-u_{0}}^{u_{0}} \ln [\tilde{M}(u)] \rho(u) d u\right\}
\end{gathered}
$$

Since the maximum eigenvalue has momentum zero it is interesting to compare equations (19)-(20) with the corresponding ones in the six-vertex model [1]. This comparison enables us to express the free energy $f(\rho)$ of our model, at a given density $\rho$, in terms of the free energy $f^{6 v}(\rho)$ of the six-vertex model, i.e.

$$
f(\rho)=\beta^{-1} \rho \ln a+(1+\rho) f^{6 v}\left(\frac{\rho}{1+\rho}\right) .
$$

We can also obtain an exactly integrable quantum Hamiltonian, related with our vertex model by taking the logarithmic derivative of the row-to-row transfer matrix (4) at $u=-\lambda$. This gives us a new integrable quantum model with Hamiltonian

$$
\begin{aligned}
H & =-N \cosh \lambda+2 \cosh \lambda \sum_{j=1}^{L} \sum_{m=1}^{\infty}(m-1) E_{j}^{m, m} \\
& +\sum_{j=1}^{L} \sum_{m, n=1}^{\infty}\left(E_{j}^{m, m+1} E_{j+1}^{n+1, n}+E_{j}^{m+1, m} E_{j+1}^{n, n+1}\right)
\end{aligned}
$$


where $E_{j}^{n, m}$ are infinite-dimensional matrices with elements given by $\left(E^{l, m}\right)_{i j}=\delta_{l, i} \delta_{m, j}$. This Hamiltonian describes the dynamics of particles on a lattice with no volume exclusion. It contains a hopping term (second sum) which allows the motion of one of the particles at a given site to its neighbouring sites, independently of its relative occupation and a static interaction (first sum) proportional to the occupation number at the site, $n_{i}=\sum_{m=1}^{\infty} m E_{i}^{m, m}$. The line conservation in the vertex model translates into the commutation of (23) with the total number of particles $n=\sum_{i=1}^{L} n_{i}$. The eigenenergies in a given eigensector with $n$ particles are given by $E_{\{k\}}=-2 \sum_{j=1}^{n} \cos k_{j}$, up to an additive constant which we have dropped, where $\left\{k_{j}\right\}$ are the solutions of (16). In the thermodynamic limit the ground-state energy is obtained in terms of the solution $\rho(u)$ of (19)-(20), namely

$$
\frac{E_{0}}{N}=2 \int_{-u_{0}}^{u_{0}}\left[\cos \mu-\frac{\sin ^{2} \mu}{\cosh u-\cos \mu}\right] \rho(u) d u .
$$

In figure 3 we show the ground-state energy per site for $0<\rho \leq 1$, obtained by solving numerically (19), (20) and (24).

To study the physical properties of this quantum chain we calculated the long-distance behaviour of the correlation functions by exploring the consequences of conformal invariance (see [15-18] and references therein). The critical fluctuations are governed by a conformal field theory with central charge $c=1$ and as in the XXZ chain the critical exponents are like those of a Gaussian model $[19,15]$. The long-distance behavior of the density-density and of the pair-pair correlation functions are given by

$$
\langle\rho(r) \rho(0)\rangle \simeq \rho^{2}+A_{1} r^{-2}+A_{2} r^{-\alpha} \cos (2 \pi \rho r)
$$

and

$$
\left\langle\eta^{+}(r) \eta(0)\right\rangle \simeq B r^{-\beta}
$$

where $\rho(r)=\sum_{m=1}^{\infty} m E_{r}^{m, m}$ and $\eta(r)=\sum_{m=2}^{\infty} E_{r}^{m-2, m}$ are the density and pair operators. The exponents $\alpha$ and $\beta$ describing the algebraic decay of the correlations are given by

$$
\alpha=\beta^{-1}=2\left[\xi\left(u_{0}\right)\right]^{2},
$$


where the dressed charge function $\xi(u)$ is obtained by solving the integral equation

$$
\xi(u)+\frac{1}{2 \pi} \int_{-u_{0}}^{u_{0}} \frac{\sin (2 \mu) \xi\left(u^{\prime}\right)}{\cosh \left(u-u^{\prime}\right)-\cos (2 \mu)} d u^{\prime}=(1+\rho) .
$$

As usual in one dimension the model does not show finite off-diagonal long-range order. However, the power decay of the pair-pair correlation (27) indicates the preference of pairing of particles whenever the exponent $\beta$ of this correlation is smaller than that of the densitydensity correlation $\alpha$ [16]. The exponent $\beta$ is plotted in figure 4 for $0<\rho \leq 1$ and some values of the parameter $\mu$. These results are obtained by solving numerically the integral equations (19-20) and (28). We see from this figure that for all values $-1 \leq \Delta=-\cos \mu<1$ there exists a regime with dominant pair correlations. The analogous behaviour of correlation functions was also observed in the models considered in Refs. [20-21]. But differently from these models, in the present case we have the nice feature that for increasing values of $\rho$, the exponent $\beta$ for $0 \leq \Delta<1$ tends toward zero and there is no regime where the density-density correlations dominate.

It should be emphasized that the formalism exposed here for creation of new integrable models applies to lattice systems with higher spin like the Perk-Shultz [8] and Sutherland models [10] as well as Fateev-Zamolodchikov [11] and Izergin-Korepin models [12].

This work was supported in part by CNPq anf FINEP-Brazil. 


\section{REFERENCES}

[1] Lieb E H 1967 Phys. Rev. 162162

[2] Yang C N and Yang C P 1966 Phys. Rev. 1150 321; 151258

[3] Baxter R J 1982 Exactly solved models in statistical mechanics (New York: Academic)

[4] Gaudin M 1983 La Fonction d'Onde de Bethe (Paris: Masson)

[5] Korepin V E, Izergin A G and Bogoliubov N M 1992 Quantum Inverse Scattering Method and Correlation Functions (Cambridge: Cambridge University)

[6] Yang C N 1967 Phys. Rev. Lett. 191312

[7] Sutherland B 1967 Phys. Rev. Lett. 19103

[8] Perk J H H and Schultz L 1981 Phys. Lett. A 84407

[9] Babelon O, de Vega H J and Viallet C M 1981 Nucl. Phys. B 190 [FS3] 542; 1982 Nucl. Phys. B 200 [FS4] 266

[10] Sutherland B 1975 Phys. Rev. B 123795

[11] Zamolodchikov A B and Fateev V 1980 Sov.J.Nucl.Phys. 32298

[12] Izergin A G and Korepin V E 1981 Commun. Math. Phys. 79303

[13] Baxter R J, Kelland S B and Wu F 1976 J. Phys. A: Math. Gen. 9397

[14] Alcaraz F C, Barber M N and Batchelor M T 1988 Ann. Phys. (N. Y.) 182280

[15] Cardy J L 1986 Nucl. Phys. B 270 [FS16] 186

[16] Bogoliubov N M and Korepin V E 1989 Int. J. Mod. Phys. B 3427

[17] Woynarovich F, Eckle H P and Truong T T 1984 J. Phys. A: Math. Gen 224027

[18] Bariev R Z, Klümper A, Schadschneider A and Zittartz J 1993 J. Phys. A: Math. Gen 26 1249; 4863; 1994 Physica B 194-196, 1417 
[19] Kadanoff L P and Brown A C 1979 Ann. Phys. (N. Y.) 121318

[20] Bariev R Z 1994 Phys. Rev. B 49 1447; Bariev R Z, Klümper A, Schadschneider A and Zittartz J 1994 Phys.Rev. B 50, 9676; Bariev R Z, Klümper A and Zittartz J 1995 Europhys. Lett. 3285

[21] Alcaraz F C and Bariev R Z 1998 Phys. Lett. A 240247 


\section{FIGURES}

FIG. 1. The vertex formed by the link variables $\alpha, \beta, \gamma, \delta$ and having Boltmann weight $R_{\gamma, \delta}^{\alpha, \beta}$.

FIG. 2. An example of a line representation of a configuration in the unlimited vertex model.

FIG. 3. A typical arrangement of lines of two adjacent rows.

FIG. 4. The ground-state energy per site $E_{0} / N$ as a function of the density $\rho=n / N$ and anisotropy $-1 \leq \Delta=-\cos \mu<1$, with (a) $\mu=\pi / 10$, (b) $\mu=\pi / 4$, (c) $\mu=\pi / 3$, (d) $\mu=\pi / 2$, (e) $\mu=5 \pi / 6$ and (f) $\mu=10 \pi / 12$.

FIG. 5. The critical exponent $\beta$ as a function of the density $\rho=n / N$, and for some values of the anisotropy $\Delta=-\cos \mu$, with (a) $\mu=\pi / 10$, (b) $\mu=\pi / 6$, (c) $\mu=\pi / 4$, (d) $\mu=\pi / 2$, (e) $\mu=5 \pi / 6$ and (f) $\mu=10 \pi / 12$. 


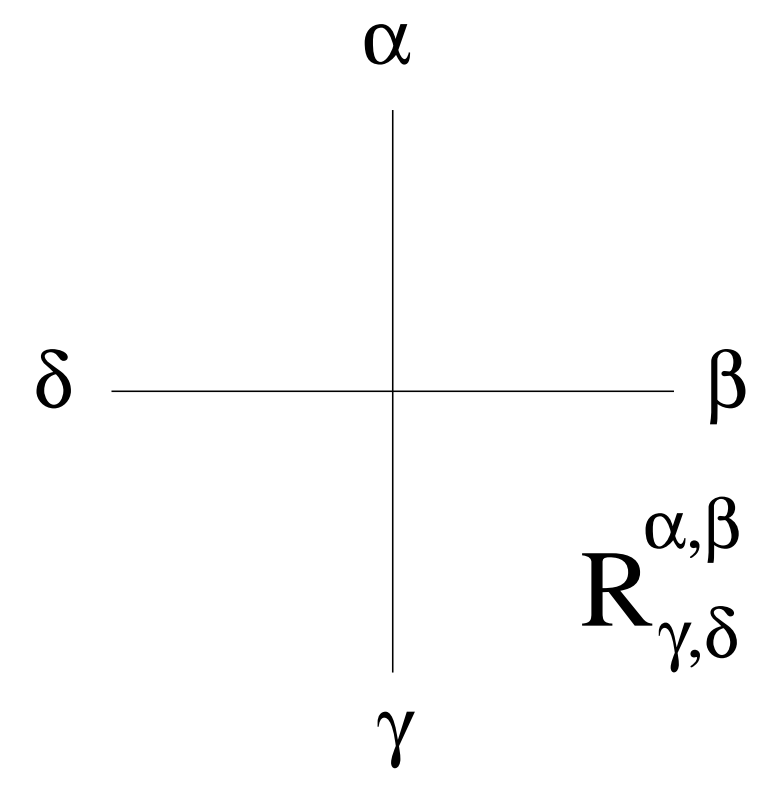




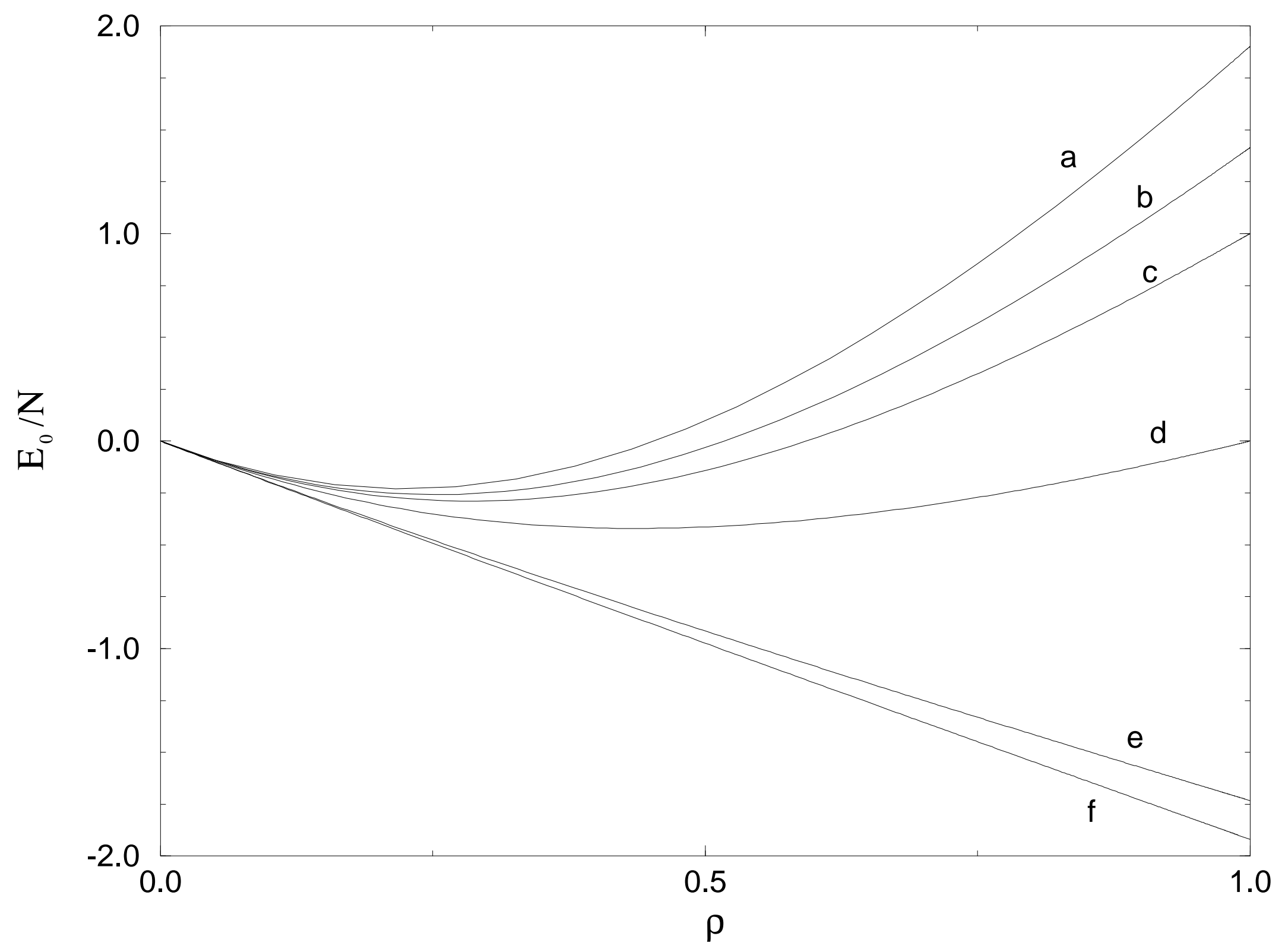




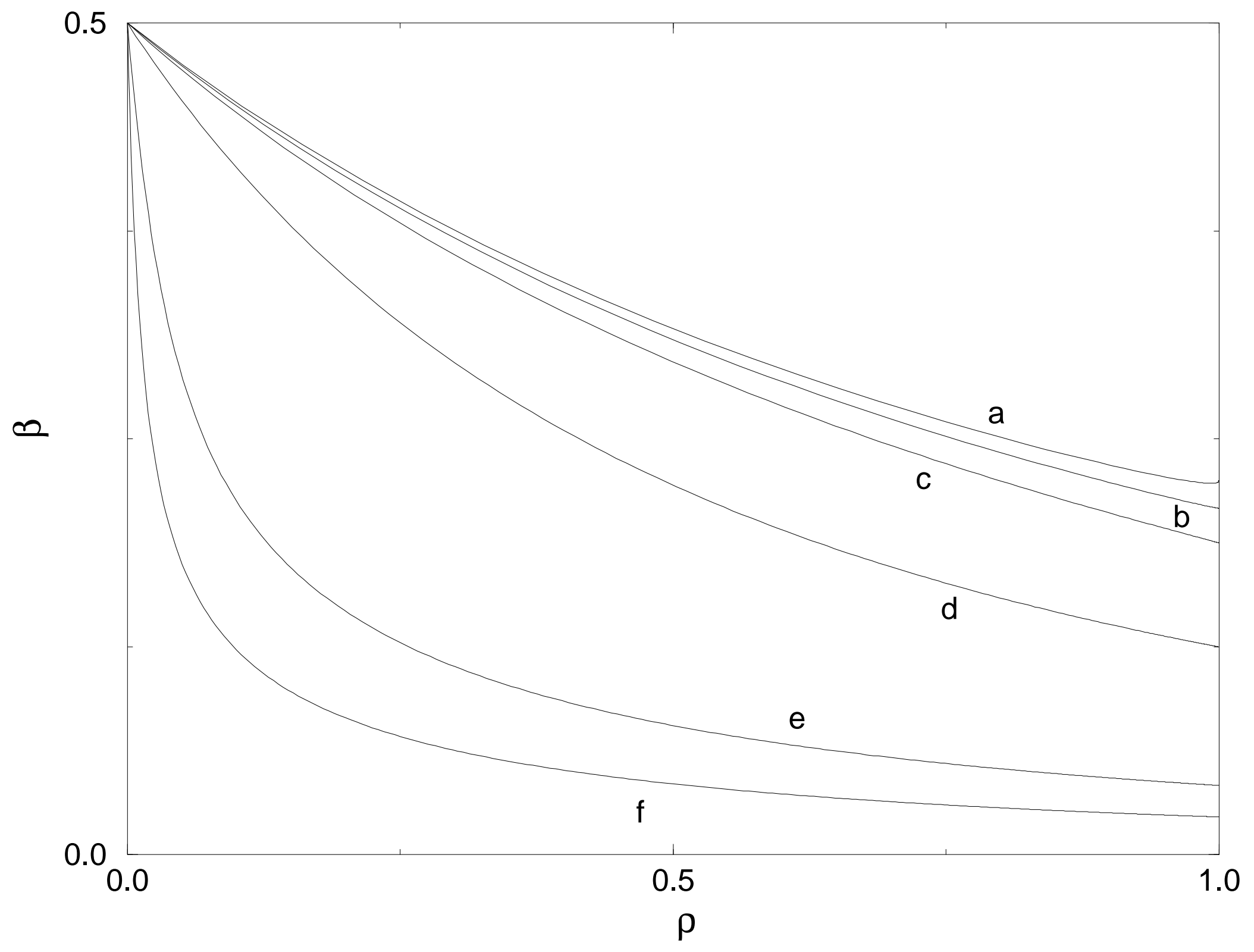

